\title{
Mitochondrial 3-Hydroxy-3-Methylglutaryl-CoA Synthase Deficiency: Clinical Course and Description of Causal Mutations in Two Patients
}

\author{
LUIGI BOUCHARD, MARIE-FRANCE ROBERT, DMITRIY VINAROV, CHARLES A. STANLEY, \\ GEOFFREY N. THOMPSON, ANDREW MORRIS, JAMES V. LEONARD, PATTI QUANT, \\ BETTY Y.L . HSU, AVIHU BONEH, YOUSSEF BOUKAFTANE, LYUDMILA ASHMARINA, \\ SHUPEI WANG, HENRY MIZIORKO, AND GRANT A. MITCHELL
}

\begin{abstract}
Service de génétique médicale, Research Center, Ste-Justine Hospital, 3175 Côte Ste-Catherine, Montreal (Québec), Canada, H3T 1C5 [L.B., M.-F.R. Y.B., L.A., S.W., G.A.M.]; Department of Biochemistry, Medical College of Wisconsin, Milwaukee, Wisconsin 53226, U.S.A. [D.V., H.M.]; The Children's Hospital of Philadelphia, Division of Endocrinology/Diabetes, Philadelphia, Pennsylvania 19104, U.S.A. [C.A.S., B.Y.L.H.]; The Murdoch Institute, Royal Children's Hospital, Melbourne 3052, Australia [G.N.T., A.B.]; Biochemistry, Endocrinology and Metabolic Unit [A.M., J.V.L.] and Paediatric Surgical Unit, [P.Q.], Institute of Child Health, London WCIN 1EH, U.K.
\end{abstract}

\begin{abstract}
Hereditary deficiency of mitochondrial HMG-CoA synthase (mHS, OMIM 600234) is a poorly defined, treatable, probably underdiagnosed condition that can cause episodes of severe hypoketotic hypoglycemia. We present clinical follow-up and molecular analysis of the two known mHS-deficient patients. The diagnosis of mHS deficiency is challenging because the symptoms and metabolite pattern are not specific. Moreover, enzyme analysis is technically difficult and requires sampling of an expressing organ such as liver. The patients, now aged 16 and $6 \mathrm{y}$, have normal development and have had no further decompensations since diagnosis. Patient 1 is homozygous for a phenylalanine-to-leucine substitution at codon 174 (F174L). Interestingly, although the F174 residue is conserved in vertebrate $\mathrm{mHS}$ and cytoplasmic HS isozymes, a Leu residue is predicted in the corresponding position of HS-like sequences from Caenorhabditis elegans, Arabidopsis thaliana, and Brassica juncea. Bacterial expression of human F174L-mHS produces a low level
\end{abstract}

mHS (EC 4.1.3.5) mediates the first and rate-limiting step of ketone body synthesis, the condensation of acetoacetyl-CoA

\footnotetext{
Received August 8, 2000; accepted September 11, 2000.

Supported by grants from the Medical Research Council of Canada, grant MT-10541 [G.M.]; National Institutes of Health (NIH), DIC 21491 [H.M].; and NIH \#1 RR 00240 [C.A.S.].

Correspondence and reprint requests: Grant A. Mitchell, M.D., Service de génétique médicale, Hôpital Ste-Justine, 3175 Côte Ste-Catherine Road, Montréal, Québec, H3T $1 \mathrm{C} 5$, Canada.

Current addresses: 220 Melbourne Street, North Adelaide, SA 5006, Australia [G.N.T.] and Department of Child Health, Royal Victoria Infirmary, Queen Victoria Road, Newcastle Upon Tyne, NE1 4L0, U.K. [A.M.].
}

of mHS polypeptide with no detectable activity. Similarly, in purified cytoplasmic HS, which in contrast to purified human mHS is stable and can be studied in detail, the corresponding $\mathrm{F} \rightarrow \mathrm{L}$ substitution causes a 10,000 -fold decrease in $V_{\max }$ and a 5 -fold reduction in thermal stability. Patient 2 is a genetic compound of a premature termination mutation, R424X, and an as-yet uncharacterized mutant allele that is distinguishable by intragenic single nucleotide polymorphisms that we describe. Molecular studies of mHS are useful in patients with a suggestive clinical presentation. (Pediatr Res 49: 326-331, 2001)
Abbreviations
ASO, allele-specific oligonucleotide
HMG-CoA, 3-hydroxy-3-methylglutaryl CoA
mHS, mitochondrial 3-hydroxy-3-methylglutaryl CoA synthase
SSCP, single-strand conformational polymorphism

and acetyl-CoA to form HMG-CoA (1). Ketone bodies are essential vectors of energy transfer from liver to extrahepatic tissues like brain, heart, and kidneys (2, 3). During fasting, ketogenesis is particularly important for the brain because circulating fatty acids, the principal noncarbohydrate energy source for most organs, are not efficiently used by the mammalian brain (4). In adult mammals, mHS is present mainly in liver (5) and testis (6).

To date, two mHS-deficient patients have been reported $(7$, $8)$. Both presented with severe hypoketotic hypoglycemia, mild 
hepatomegaly, or fatty liver, and a nondiagnostic pattern of urinary organic acids with the increase of medium and short chain dicarboxylic acids, suggestive of a fatty acid oxidation disorder. Liver mHS activity was deficient in both patients. To date, the diagnosis of mHS deficiency has relied only upon enzyme assay.

mHS is a $57 \mathrm{kD}$ mitochondrial matrix homodimer (6). Human mHS cDNAs and the human mHS gene have been cloned (9-11). The human mHS gene spans $25 \mathrm{~kb}$ and contains 10 exons. Its locus, HMGCS2, is on chromosome 1p12-13 (9) between markers WI-7519 and D1S514 (12). In this article, we report pathogenic mutations in both patients, as well as four mHS gene polymorphisms and a prokaryotic expression system for recombinant mHS.

\section{MATERIALS AND METHODS}

Clinical samples. Patients 1 (7) and 2 (8) have been described. Genomic DNA from both patients and their parents was available, as was a needle biopsy sample from the liver of Pt 1. Control liver RNA for mHS cDNA synthesis was from a patient with an unrelated disease (hepatorenal tyrosinemia). Informed consent for molecular diagnostic testing of mHS was obtained from the families.

Amplification and mutation detection. Mutation detection was performed with DNA isolated from patients, parents, and controls using amplification with flanking intronic primers for mHS exons 1-9 followed by SSCP analysis and, in some cases, sequence analysis. The PCR conditions were as follows. In a $50-\mu \mathrm{L}$ reaction, the indicated amounts of each primer and 100 ng of genomic DNA were added to a mixture containing a final concentration of dNTP, each $12.5 \mu \mathrm{M} ;{ }^{35} \mathrm{~S}-\mathrm{dATP}$ and ${ }^{35} \mathrm{~S}$ dCTP (Amersham Pharmacia Biotech, Baie d'Urfé, Québec, Canada), $12.5 \mu \mathrm{Ci}$ each; Taq DNA polymerase (GIBCO-BRL, Burlington, Ontario, Canada), $2.5 \mathrm{U}$; Tris- $\mathrm{HCl}$ ( $\mathrm{pH} 8.4$ ), 20 $\mathrm{mM} ; \mathrm{KCl}, 50 \mathrm{mM}$, and the indicated concentration of $\mathrm{MgCl}_{2}$. Following a hot start, PCR amplification cycles were performed as follows. The details of the amplification are presented as follows for each exon: sequence of the $5^{\prime}$ primer, pmol of $5^{\prime}$ primer used in the reaction; 3 ' primer sequence, pmol; times (s) for denaturation at $95^{\circ} \mathrm{C}$-annealing (temperature, ${ }^{\circ} \mathrm{C}$ )-extension at $72^{\circ} \mathrm{C}$; cycle number; magnesium concentration $(\mathrm{mM})$ and amplicon length (bp). Exon 1, GCAGGAGTCTTGGGGCTT, 10; CTAGTTACAAGGTGCTTCTC, $10 ; 30-30\left(59^{\circ} \mathrm{C}\right)-30 ; 30 ; 1.5 ; 243$. Exon 2 : TGATTGCTATGTTCATGTCTT, 15; TCAGCCTCCAATTCCAGCACC, 15; $30-30\left(62^{\circ} \mathrm{C}\right)-20,30 ; 1.5 ; 585$. Exon 3 : TATACCCCAGGACCTAGAA, 50; GGTGTGCCAATGTGGGA, 50; $30-30\left(59^{\circ} \mathrm{C}\right)-20 ; 40 ; 2.0 ; 285$. Exon 4: TCTCTCATCTTTTGTGCT, 15; CCCCACTTTCTGCCCTC, $15 ; 30-20\left(60^{\circ} \mathrm{C}\right)$ 20; 30; 1.5; 263. Exon 5: GAGGATATCTAAATGGCT, 50; TTCTTGCTCACACCTCAC, $50 ; 30-30\left(58^{\circ} \mathrm{C}\right)-20 ; 30 ; 1.0$; 277. Exon 6: GTCCTCTCCAGAAGCTGT, 50; TGAGGGTGTGCATGGAGG, $50 ; 40-30\left(64^{\circ} \mathrm{C}\right)-30 ; 30 ; 1.5 ; 302$. Exon 7: CCACTCTGCCCAAGAACT, 50; CCTCAGTAATGGTGGGGAA, $50 ; 30-30\left(58^{\circ} \mathrm{C}\right)-20 ; 40 ; 1.0 ; 188$. Exon 8, CTCGTGATTGGCAATAGT, 50; TGGGGTCTCTCTTCCTC, $50 ; 40-30\left(64^{\circ} \mathrm{C}\right)-30 ; 30 ; 2.0 ; \approx 200$. (The length is approximate because of a variable microsatellite within the amplicon (12)). Exon 9: GTACTGCCTCTTCTCCTCATT, 50; TTCTTCTCCCTGCACCTGTCC, 50: $40-30\left(64^{\circ} \mathrm{C}\right)-30$; $30 ; 1.0 ; 242$. The last cycle concluded with a 10 -min extension at $72^{\circ} \mathrm{C}$.

SSCP was performed using three different gel conditions as described previously (13). Abnormally migrating fragments were analyzed by direct sequencing (Thermo Sequenase, Pharmacia, Biotech Inc., Baie d'Urfé, Québec, Canada). Special considerations apply to exons 2 and 8 . Before electrophoresis, the long exon 2 amplicon was digested with Nco I, which cleaves at cDNA residue 358 . The exon 8 amplicon contains a highly polymorphic microsatellite (12) and was sequenced without SSCP.

ASO hybridization. To screen for mutations in normal controls in exons 1, 2, 7 and 9 (see "Results"), genomic DNA samples were amplified and the reaction products were slot blotted and hybridized to radiolabeled ASO. Oligonucleotides were end-labeled using $\gamma^{32} \mathrm{P}-\mathrm{ATP}$ and $5^{\prime}$ nucleotide kinase as recommended by the supplier (GIBCO-BRL). Hybridization was as described (14). The oligonucleotides, their orientations, and their positions in the mHS cDNA were as follows. The exon 1 normal sequence oligonucleotide is $5^{\prime}$-CTGCTTGCTCCTCTGG-3', sense strand, hybridization temperature $35^{\circ} \mathrm{C}$; washing temperature $47^{\circ} \mathrm{C}$. The exon 1 mutant sequence oligonucleotide is 5'-CTGCTTGTTCCTCTGG-3', sense, hybridization $35^{\circ} \mathrm{C}$; washing $49.5^{\circ} \mathrm{C}$. The exon 2 normal sequence (F174F) oligonucleotide is $5^{\prime}$-CTCCCTCTTCAATGCT-3' sense, hybridization $35^{\circ} \mathrm{C}$; washing $22^{\circ} \mathrm{C}$. The exon 2 mutant sequence (F174L) oligonucleotide is $5^{\prime}$-CTCCCTCCTCAATGCT-3', sense, hybridization $35^{\circ} \mathrm{C}$; washing $22^{\circ} \mathrm{C}$. The exon 7 normal sequence (R424R) oligonucleotide is $5^{\prime}$-TTCATTTCGAGTATCC-3', sense, hybridization $35^{\circ} \mathrm{C}$; washing $47^{\circ} \mathrm{C}$. The exon 7 mutant sequence ( $\mathrm{R} 424 \mathrm{X}$ ) oligonucleotide is $5^{\prime}$ TTCATTTTGAGTATCC-3', sense, hybridization $35^{\circ} \mathrm{C}$; washing $44^{\circ} \mathrm{C}$.

cDNA synthesis from patient 1. cDNA fragments were amplified following reverse transcription from $1 \mu \mathrm{g}$ of total liver RNA isolated from patient 1 and from controls, using the Superscript II kit (Pharmacia) as described by the manufacturer.

Bacterial expression of recombinant mature human mHS cDNAs. Priming was performed from the liver RNA template of patient 1, using 5'-AAAATTTGGAGATTCACAGGAACAG-3', complementary to nucleotides 1943-1919 in the 3' untranslated region. PCR was performed in $50 \mu \mathrm{L}$ containing $1 \mu \mathrm{L}$ of the above reverse transcription reaction; $100 \mathrm{ng}$ of each primer (see below); dNTPs, each $12.5 \mu \mathrm{M}$; Tris $\mathrm{HCl}$ ( $\mathrm{pH}$ 8.4), $20 \mathrm{mM}$; KCl, $50 \mathrm{mM} ; \mathrm{MgCl}_{2}, 2.0 \mathrm{mM}$; Taq polymerase (GIBCO-BRL), 2.5 U. Following a hot start, 30 PCR amplification cycles were performed as follows: $95^{\circ} \mathrm{C}, 30 \mathrm{~s} ; 60^{\circ} \mathrm{C}$, $30 \mathrm{~s} ; 72^{\circ} \mathrm{C}, 90 \mathrm{~s}$; followed by a final extension for $10 \mathrm{~min}$ at $72^{\circ} \mathrm{C}$. The primers were: sense 5'-AAGAATTCACAGCCTCTGCTGTCCC-3' containing a cloning EcoRI site and mHS nucleotides 112-128; and antisense 5'-AAGGATCCTCTCCATTGCTCCATC-3' containing a cloning BamH1 site and mHS nucleotides 1789-1774. The amplified fragment contains mHS cDNA nucleotides 112-1789, and includes the complete 
coding sequence of mature mHS. The normal and mutant cassettes were sequenced on each strand.

We used an expression system in Escherichia coli DH5 $\alpha$ based on the pTRC 99a vector (Pharmacia). The mHS cDNA was inserted between the EcoRI and BamH1 sites. This vector contains mHS cDNA residues corresponding to amino acids 38-471 of normal and mutant (F174L) mHS, plus the amino terminal tripeptide Met-Glu-Phe. We induced log phase cultures by incubation in the presence of $1 \mathrm{mM}$ IPTG for $9 \mathrm{~h}$. Bacteria were isolated by centrifugation at $6000 \mathrm{~g}$ for $10 \mathrm{~min}$. To obtain crude lysates, cells were placed in lysis buffer (Tris- $\mathrm{HCl} \mathrm{pH} 8.0,10 \mathrm{mM} ; \mathrm{CaCl}_{2}, 10 \mathrm{mM}$; DTT, $1 \mathrm{mM}$; EDTA, $1 \mathrm{mM}$; phenylmethylsulfonyl fluoride (PMSF), 0.1 $\mathrm{mM}$; glycerol, 10\%) then sonicated with a sonic dismembrator (Artek Systems Corporation, Farmingdale, NY, U.S.A.) twice for 15 secs each, at power setting 60 , separated by a 30 -s period on ice, followed by centrifugation at $13,000 \mathrm{~g}$ for $10 \mathrm{~min}$. In some experiments, mHS-containing plasmids were coexpressed with pGroESL (generously supplied by A. Gatenby, DuPont Experimental Station, Wilmington, DE, U.S.A.), which expresses the chaperonins GroEL and $\operatorname{GroES}(15,16)$.

mHS assay and Western blot analysis. $\mathrm{mHS}$ activity is followed by monitoring the decrease in absorbance at $300 \mathrm{~nm}$ of the magnesium chelate of acetoacetyl-CoA (17). The complete assay mixture contains $100 \mathrm{mM}$ Tris-HCl, $\mathrm{pH} 8.2 ; 0.1$ mM EDTA; $20 \mathrm{mM} \mathrm{MgCl} 2 ; 60 \mu \mathrm{M}$ acetoacetyl-CoA and 0.3 $\mathrm{mg}$ of lysate protein in a total volume of $1.0 \mathrm{~mL}$. The reaction is initiated by the addition of acetyl-CoA, $400 \mu \mathrm{M}$, and is linear for at least $5 \mathrm{~min}$ at $30^{\circ} \mathrm{C}$. The apparent extinction coefficient for acetoacetyl-CoA under the assay conditions is $12,200 \mathrm{M}^{-1} \mathrm{~cm}^{-1}$. One unit of HS is defined as the amount that catalyzes the disappearance of $1 \mu \mathrm{mol}$ of acetoacetate per min at $30^{\circ} \mathrm{C}$ under the conditions described. Protein determination was as described (18).

Rabbit polyclonal antibodies against recombinant human mHS were prepared as follows. A 1678 bp human mHS cDNA fragment spanning amino acids 38-508 was obtained by reverse-transcription PCR amplification of human liver RNA, inserted into the pET-30a vector (NOVAGEN Inc., Madison, WI, U.S.A.) restricted with BamH1 and EcoRI, and sequenced on both strands. The plasmid was expressed in E. coli strain BL21(DE3) pLysS. The mHS/poly-His fusion protein was purified by affinity chromatography on a HisBind resin column (Novagen) and used to immunize a New Zealand white rabbit. Antibody was purified by passage through a $\mathrm{CNBr}-\mathrm{Sepharose}$ (Pharmacia) column coupled with lysate proteins from bacteria expressing the pET-30a plasmid with no insert. Western blotting was performed at a dilution of 1:30,000.

Northern blots. Ten micrograms of total RNA were resolved in an agarose gel and transferred to a nylon membrane (MSI). After a 4-h prehybridization in hybridization buffer [SSPE 5X $\left(\mathrm{NaH}_{2} \mathrm{PO}_{4}, 0.05 \mathrm{M}\right.$; EDTA, $5 \mathrm{mM}$; NaCl, $0.9 \mathrm{M}$, pH 7.7); formamide, 50\%; Denhardt's 5X (Ficoll type 400, 0.1\%; polyvinylpyrrolidone, $0.1 \%$; BSA fraction $\mathrm{V}, 0.1 \%$ ); dextran sulfate, $10 \%$ ], hybridization was carried out at $42^{\circ} \mathrm{C}$ with a radiolabeled Bam H1-EcoRI mHS cDNA fragment (nucleotides 112-1789), labeled with the Random Prime DNA kit (Pharmacia Biotech Inc., Baie d'Urfé, Québec, Canada). Filters were washed in $2 \mathrm{X} \mathrm{SSC}(\mathrm{NaCl}, 0.3 \mathrm{M}$; sodium citrate, $0.03 \mathrm{M}$, $\mathrm{pH} 7.0) / 0.1 \%$ SDS at room temperature. Following exposure, the membrane was reprobed with a human HL cDNA fragment (nucleotides -14-1575) as a control for RNA quality and quantity.

Southern blotting. Southern blotting of genomic DNA was performed as described (19) using the full-length coding sequence mHS cDNA as probe (nucleotides 1-1789).

Expression and purification of mutant cytosolic HS (cHS). We substituted a Leu codon for Phe at position F137 in cHS, which corresponds to F174 in mHS. We used the QuickChange Site-Directed Mutagenesis Kit (Stratagene, La Jolla, CA, U.S.A.) and the following primers (Oberon, Alameda, CA, U.S.A.): 5'-GC-TAT-GGA-GGC-ACT-GCT-GCT-CTT-TTAAAT-GCT-ATT-AAC-TGG-3' and 3'- CG-ATA-CCT-CCGTGA-CGA-CGA-GAA- $A A T$-TTA-CGA-TAA-TTG-ACC-5'. The mutagenic codons are underlined. F137L was engineered into wild-type chicken cHS cDNA encoded in the pACS plasmid (20). The incorporation of F137L as well as the absence of any PCR artifacts in the coding sequence was confirmed by DNA sequencing. The resulting plasmid was transformed into E. coli BL21(DE3) and the mutant protein was expressed upon induction with IPTG $(1 \mathrm{mM})$. Bacteria were harvested and disrupted using a French pressure cell. The soluble protein fraction was prepared and subjected to ammonium sulfate fractionation and anion exchange chromatography (Pharmacia Fast Q, Pharmacia Biotech Inc., Piscataway, NJ, U.S.A.) as described (20). Eluate containing recombinant cHS was dialyzed against $5 \mathrm{mM}$ potassium phosphate buffer $(\mathrm{pH}$ 7.0) containing $0.1 \mathrm{mM}$ EDTA and $0.1 \mathrm{mM}$ DTT and loaded onto a $1 \times 20 \mathrm{~cm}$ hydroxylapatite-HTP column equilibrated with the same buffer. A $300-\mathrm{mL}$ gradient $(5-170 \mathrm{mM}$ potassium phosphate $(\mathrm{pH}$ 7.0) containing $0.1 \mathrm{mM}$ EDTA and 0.1 $\mathrm{mM}$ DTT) was used to elute the enzyme. Pooled fractions were concentrated and chromatographed on a Superose-6HR FPLC column, equilibrated with $50 \mathrm{mM}$ potassium phosphate $(\mathrm{pH}$ 7.2) containing $100 \mathrm{mM} \mathrm{NaCl}$. Homogeneous enzyme was recovered, brought to $20 \%$ glycerol, and stored at $-80^{\circ} \mathrm{C}$. From a 2-L bacterial culture, $38 \mathrm{mg}$ of mutant cHS were isolated. For comparison, about $80 \mathrm{mg}$ of wild-type cHS can be obtained from a similar volume of culture medium.

Kinetic characterization and stability measurements of mutant cHS. The low residual activity of the mutant cHS required use of a sensitive radioisotopic assay (17) for all steady state kinetic measurements. For determination of $V_{\max }$ and $\mathrm{K}_{\mathrm{m}}$, assays were performed at a concentration of acetoacetyl-CoA of $20 \mu \mathrm{M}$ and of ${ }^{14} \mathrm{C}$ acetyl-CoA ranging from 5 to $100 \mu \mathrm{M}$. $V_{\max }$ and $\mathrm{K}_{\mathrm{m}}$ values were determined by nonlinear regression fits to the Michaelis-Menten equation. For stability measurements, wild-type or mutant cHS was incubated at $40^{\circ} \mathrm{C}$ in $100 \mathrm{mM}$ Tris-Cl buffer (pH 8.2) and aliquots withdrawn for assay at $0,5,10,20,40,60$, and $90 \mathrm{~min}$. Data were plotted as $\log \%$ initial activity versus time; the reported rates of decay represent optimal fits of the data using the program Table Curve 2D (Levenburg-Marquardt algorithm, SPSS Science, Chicago, IL, U.S.A.). 


\section{RESULTS AND DISCUSSION}

Patient follow-up. Both patients are developing normally and have had no further episodes of hypoglycemic decompensation. Patient 1 is now 16 y old, has a normal physical examination, has reached the Tanner 5 stage of sexual development, performs normally at school, and is active in sports. He typically fasts for up to $16 \mathrm{~h}$ with no symptoms. On the rare occasions when he becomes ill, he has ingested a carbohydraterich supplement $(30 \% \mathrm{wt} / \mathrm{vol})$. His parents have no known consanguinity. Patient 2 is now 6 y old and has been healthy since the original report.

SSCP analysis. We found subtle migration abnormalities in exon 2 of patient 1 (not shown). There was also a very subtle change in the migration of exon 7 of patient 2 (not shown), and marked differences in exon 1 of patient 1 and in exon 9 of both patients and one control (not shown). The differences in exons 1 and 9 were due to variants in noncoding regions (described below).

Mutations in the mHS coding sequence. The migration abnormality of exon 2 of patient 1 was due to a $\mathrm{T} \rightarrow \mathrm{C}$ transition at nucleotide 520 that changes the normal Phe 174 codon (TTC) to Leu (CTC) and is designated F174L (Fig. 1A). In exon 7 of patient 2 we found a $\mathrm{C} \rightarrow \mathrm{T}$ transition in a $\mathrm{CpG}$ pair at nucleotide 1270, converting the normal Arg 424 codon (CGA) to a stop codon (TGA) and designated R424X (Fig. 1B). We screened normal controls for the presence of F174L (Fig. 2, upper panel) and R424X (Fig. 2, lower panel) using allelespecific oligonucleotides. None carried F174L (0/106 alleles, 72 of Caucasian and 34 of Asian origin, 22 of which were of Chinese origin) or R424X (0/84 alleles of diverse ethnic origins). By direct sequencing and ASO analysis, patient 1 is homozygous for F174L and his parents are heterozygous. Patient 2 and his father are heterozygous for R424X whereas by ASO analysis his mother is normal at codon 424 .

We subsequently amplified and sequenced all of the exons and flanking sequences of both patients and performed genomic Southern analysis with several restriction enzymes in patient 2 . No other point mutations were detected in the coding sequence and the Southern blots were normal in patient 2 .

Functional characterization of F174L. F174 is located in a highly conserved region of mHS, 8 residues downstream from the catalytic cysteine residue. However, after alignment of reported HS and HS-like species (Fig. 3), the predicted sequences from Caenorhabditis elegans and two plants (Arabidopsis thaliana and Brassica juncea) have a leucine in the position corresponding to F174 in human mHS. Although none of these sequences have been studied functionally, the presence of a Leu codon in three organisms prompted us to sequence the rest of the mHS coding sequence from patient 1 rather than presuming that $\mathrm{F} 174 \mathrm{~L}$ was the causal mutation. Because the rest of the sequence was normal, we studied the F174L mutation directly.

We designed a bacterial expression system to test $\mathrm{mHS}$ mutants. mHS activity in cells expressing the F174L plasmid was $0.05 \pm 0.40 \mathrm{mU} / \mathrm{mg}$ protein (mean $\pm \mathrm{SE}, n=7$ assays), compared with $6.78 \pm 0.81 \mathrm{mU} / \mathrm{mg}$ protein for the normal plasmid (range 5.77 to $8.20, n=8$ ). To test whether mutations
A
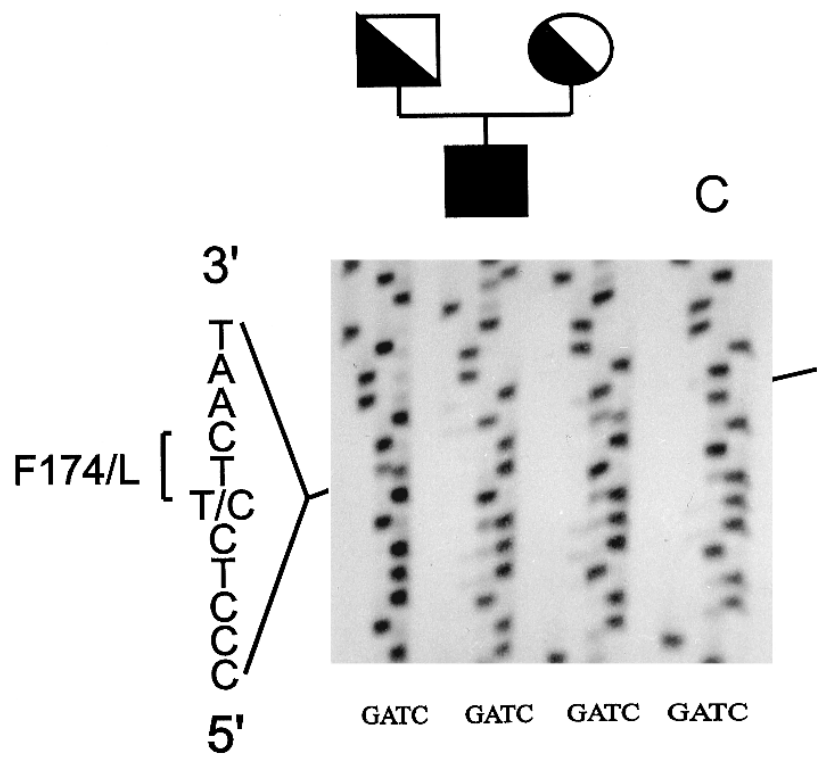

$\mathbf{B}$

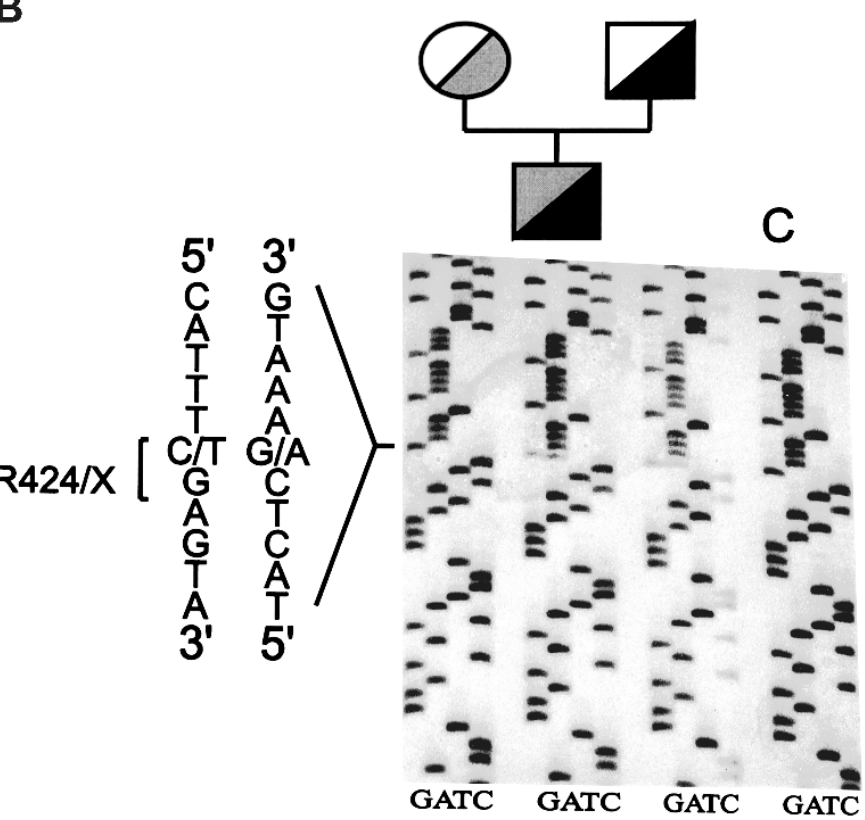

Figure 1. Mutations in the coding sequences of patients 1 and 2. Direct sequencing of normal and mutant genomic DNA is shown. (A) Exon 2, family 1, showing the $\mathrm{T} \rightarrow \mathrm{C}$ transition at nucleotide 520 (F174L). (B) Exon 7, family 2 , showing the $\mathrm{G} \rightarrow \mathrm{A}$ transition in the antisense strand corresponding to R424X.

that suppressed mHS expression had occurred elsewhere in the vector than at position 174 , we replaced the mutant $\mathrm{mHS}$ cassette with a normal sequence. This construct produced normal mHS activity (not shown).

Coexpression of mHS cDNAs with GroESL chaperonins (Fig. 4) increased the level of wild-type mHS and also raised the level of F174L-containing mHS to detectable levels. Some mHS peptide is detectable even in noninduced cells, presumably reflecting leaky expression under uninduced conditions. Of note, a higher level of mHS peptide was present in IPTGinduced cells expressing F174L-mHS than in non-IPTGinduced cells expressing normal mHS (Fig. 4). In contrast, 

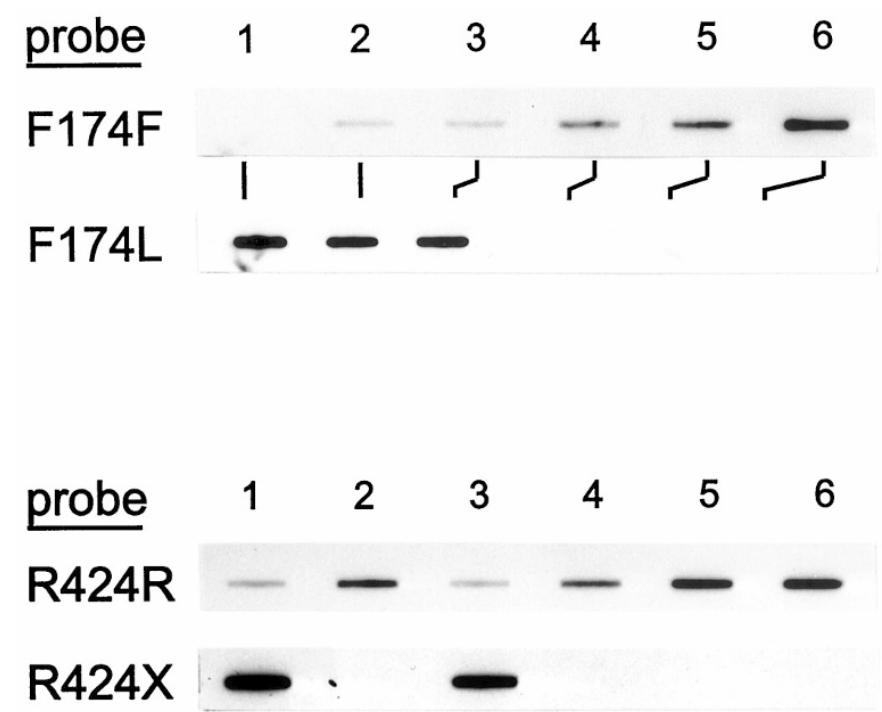

Figure 2. ASO testing at mHS codons 174 and 424. Upper panel, wild type (F174F) and F174L. Positions in the array are as follows: 1, patient 1; 2, father of patient $1 ; 3$, mother of patient $1 ; 4-6$, normal controls. Lower panel, R424R and R424X: 1, patient 2;2, mother of patient 2; 3, father of patient 2; 4-6, normal controls. No signal was detectable in blank lanes containing no DNA (not shown).

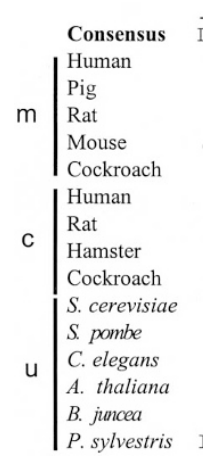

\begin{tabular}{ccccc}
\multicolumn{1}{c}{ LEE. SGNTD. } & \multicolumn{4}{c}{$*$} \\
D & I & I & T & \\
D & I & I & T & \\
D & I & I & T & \\
D & I & I & T & \\
E & V & I & I & R \\
E & I & I & T & \\
E & I & I & T & \\
E & I & I & T & \\
KD-- & I & V & N & \\
GE-- & V & I & L & \\
GD-NHNV & I & CV & \\
$--P$ & S I & V & IK & F \\
KC & V & V S & & \\
EKC & V & V & S & \\
KC & EI & V S & &
\end{tabular}

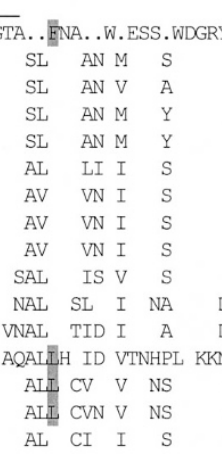

$\begin{array}{cll}\text { M } & \text { C } & \text { \#U81860 } \\ \text { V } & \text { C } & \text { \#U90884 } \\ \text { L } & \text { C } & \text { \#M33648 } \\ \text { L } & \text { C } & \text { \#B55729 } \\ \text { I } & \text { AA } & \text { \#X77516 } \\ \text { L } & \text { A } & \text { \#L25798 } \\ \text { L } & \text { A } & \text { \#X52625 } \\ \text { L } & \text { A } & \text { \#L00334 } \\ \text { L } & \text { A } & \text { \#X73679 } \\ \text { D I } & \text { C } & \text { \#X96617 } \\ \text { DGI } & \text { A } & \text { \#P54874 } \\ \text { N I } & \text { VA } & \text { \#U12787 } \\ \text { GL } & \text { IC } & \text { \#U79160 } \\ \text { GL ICT } & \text { \#AF148847 } \\ \text { GL } & \text { AT } & \text { \#X96386 }\end{array}$

Figure 3. Alignment of reported HS and HS-like DNA sequences around mHS residue 174. The consensus sequence shows residues identical in all vertebrate mHS and cHS sequences. The active site cysteine residue is indicated by an asterisk, and the position of residue 174 is shaded. The residues included in the active site peptide isolated from chicken mHS are overscored. The classes of HS ( $m$, mitochondrial; $c$, cytoplasmic; $u$, undefined) are shown, as are the species of origin and GenBank reference number. Dashes indicate deletions.

mHS activity was easily measurable in the wild-type mHS lysate $(0.28 \mathrm{mU} / \mathrm{mg})$ but was undetectable in all lysates expressing F174L mHS.

$\mathrm{mHS}$ is a labile polypeptide, perhaps because of the presence of multiple proline-, glutamate-, serine-, and threonine-rich (PEST) sequences, that are frequent in proteins with rapid turnover (21). The rapid suppression of ketogenesis following insulin or glucose administration (reviewed in (20)) may also reflect rapid protein turnover. The in vitro instability of $\mathrm{mHS}$ poses a practical barrier to detailed enzymatic characterization. Cytoplasmic HS (cHS), in contrast, is more stable than mHS, but its catalytic efficiency and all other known active site characteristics tested are equivalent to those of mHS (20). We used it as a surrogate to mHS to test F174L in a purified protein. We introduced the analogous mutation, F137L, into a

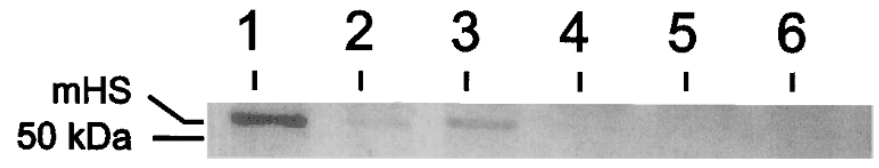

Figure 4. Western blot showing coexpression of normal or mutant mHS cDNAs and GroESL. Ten micrograms of bacterial lysate protein were loaded in each lane. 1, IPTG-induced normal mHS; 2, uninduced normal mHS; 3, IPTG-induced F174L mHS; 4, uninduced F174L mHS; 5, vector without insert, induced; and 6, without insert uninduced. The position of mHS is indicated.

cHS cDNA. During purification, F137L-cHS was not recovered from anion exchange (Fast Q Sepharose, Pharmacia Biotech Inc., Piscataway, NJ, U.S.A.) at the same high level of purity as the wild-type cHS. This necessitated additional hydroxylapatite and molecular sieve chromatography steps to obtain homogeneous peptide. We observed a reduced $\mathrm{K}_{\mathrm{m}}$ for acetyl-CoA (10.7 $\mu \mathrm{M}$ for F137L cHS compared with $290 \mu \mathrm{M}$ for wild-type) that may suggest weaker inhibition by the cosubstrate, acetoacetyl-CoA. Importantly, the measured $V_{\max }$ $(0.16 \mathrm{mU} / \mathrm{mg})$ of purified F137L-cHS is four orders of magnitude less than in wild type, reflecting a diminution in catalytic efficiency that can account for depressed activity measured in tissue samples. In addition to accounting for catalytic deficiency, purified F137L-cHS is less stable than wild-type cHS. Purified mutant cHS exhibits a 5.5 -fold diminution in $\mathrm{t}_{1 / 2}$ at $40^{\circ} \mathrm{C}$ [59 versus $336 \mathrm{~min}$ for wild-type cHS, (not shown)].

Therefore, F174L in mHS and the corresponding mutation in cHS both caused a lower level of peptide and a reduced specific activity compared with the wild-type peptides. We conclude that F174L alone is sufficient to cause the mHS deficiency of patient 1 .

F174L illustrates the limits of interspecific sequence comparisons as a method of deducing functional information about peptides. The HS-like cDNA sequences from $C$. elegans, $A$. thalinana, and $B$. juncea, that contain a Leu codon in homologous positions to F174 in mHS, merit direct functional testing. Perhaps these HS peptides contain counterbalancing amino acid substitution(s) that increase HS activity and/or stability. Alternatively, perhaps a low level of HS activity is sufficient or required in these organisms or the peptides have other functions distinct from those of HS.

The premature termination mutation in patient 2 would generate a peptide truncated by 84 amino acids and predicted to be unstable and/or inactive. Interestingly, Western blots of liver from patient 2 revealed no immunoreactive species of 52 $\mathrm{kD}$ or of lower molecular weight although substantial $\mathrm{mHS}$ activity was detectable in the assay system used (8). Because of this unusual biochemical phenotype, characterization of the patients other mHS allele will be of interest.

Noncoding mutations in the $\mathbf{m H S}$ gene. The migration abnormality detected in exon 1 was caused by a $\mathrm{C} \rightarrow \mathrm{T}$ transition at nucleotide -11 in the $5^{\prime}$ untranslated region $(\mathrm{T}(-11))$. We screened normal controls for the presence of $T(-11)$. No carriers were found in 35 Caucasian subjects tested, but 5 of 9 Chinese controls were heterozygous (27.7\% of Chinese chromosomes). A single individual of Inuit origin was also tested and is heterozygous for $\mathrm{T}(-11)$. 
In intron 9, we sequenced seven DNA samples: both patients, their parents, and a control sample that also had an unusual migration pattern. We found three point mutations: IVS $9+16$ ins $\mathrm{C}$, IVS $9+53 \mathrm{G} \rightarrow \mathrm{A}$ and IVS $9+68 \mathrm{~A} \rightarrow \mathrm{G}$. IVS $9+16$ ins $C$ was present in all samples sequenced and likely is the normal sequence at that position, with the previously-reported sequence possibly representing a rare variant. At IVS $9+53 \mathrm{G} \rightarrow \mathrm{A}$, patient 1 and his mother were homozygous $\mathrm{G}$, whereas the father of patient 1 , patient 2 and his parents, and the control were heterozygous. All three residues are outside of the conserved motif known to be important for splicing and therefore presumably lack functional significance. We have not tested this directly, nor have we tested their distribution in larger population samples.

Diagnosis of $\boldsymbol{m H S}$ deficiency. At present, there is no simple and reliable way to diagnose mHS deficiency, which probably explains why only two cases have been reported. Clinically, the symptoms of mHS deficiency are intermittent and may be subtle, and the full clinical spectrum is unknown. Hypoketotic hypoglycemia is a risk factor for neurologic damage. $\mathrm{mHS}$ deficiency is treatable, as illustrated by the course of both known patients who have been asymptomatic since diagnosis. This is consistent with the decrease with age of fatty acid flux and ketogenic rate, and the potential for efficient treatment of mHS deficiency by avoidance of fasting and by carbohydrate administration at times of lipolytic stress [reviewed in (21)]. Taking these observations together, it seems important to consider and to diagnose this condition.

Metabolite assays, the mainstay of diagnosis for most inborn errors of organic acid metabolism, are nonspecific in mHS deficiency. A tentative diagnosis of mHS deficiency can be proposed in patients whose clinical presentation suggests a fatty acid oxidation disorder (fatty liver, hypoketotic hypoglycemia, dicarboxylic aciduria) but in whom a nondiagnostic pattern of plasma acylcarnitines is present. To date, a definitive diagnosis of mHS deficiency has only been possible enzymatically. Enzyme assay for mHS is invasive, requiring biopsy of an expressing tissue such as liver. Furthermore, mHS assays are fraught with methodological problems because enzymes acting upon both of its substrates and its product are present at activity levels higher than those of mHS (21). In whole tissue homogenates, cytoplasmic HS contributes background interference, and thus the true extent of mHS deficiency can only be evaluated approximately.

Molecular analysis of mHS is a useful addition to the above techniques. It does not require access to an expressing tissue, is predicted to detect most pathologic mutations, and provides definitive evidence of mHS deficiency if a pathogenic mutation is documented. The limits of mutation testing for $\mathrm{mHS}$ are also illustrated by this report. Our initial results suggest that mHS deficiency will be caused by a variety of mutations, so screening for common mutations will not be appropriate. In both patients reported, complete sequencing of exons and flanking intronic sequences was necessary. Such techniques of mutation detection and proving the pathogenicity of missense mutations are labor-intensive. As illustrated by patient 2 , some deleteri- ous mutations are not detected by current methods. Therefore, the finding of a negative molecular result must be weighed against the clinical and biochemical evidence in favor of the diagnosis. Conversely, for confirmed cases like patient 2 , in whom a mutation is not detectable on one allele, the intragenic markers described in this article or reported previously (12) could be used for noninvasive presymptomatic diagnosis of other family members.

Acknowledgments. The authors thank Ronald Wanders for long-term collaboration and Raffaela Ballarano and Ginette Richard for secretarial assistance.

\section{REFERENCES}

1. Mitchell GA, Kassovska-Bratinova S, Boukaftane Y, Robert M-F, Wang SP, Ashmarina L, Lambert M, Lapierre P, Potier E 1995 Medical aspects of ketone body metabolism. Clin Invest Med 18:193-216

2. Robinson AM, Williamson DH 1980 Physiological roles of ketone bodies as substrates and signals in mammalian tissues. Physiol Rev 60:143-187

3. Williamson DH, Bates MW, Krebs HA 1968 Activity and intracellular distribution of enzymes of ketone-body metabolism in rat liver. Biochem J 108:353-361

4. Partridge WM 1983 Brain metabolism: a perspective from the blood-brain barrier. Physiol Rev 63:1481-1535

5. Clinkenbeard KD, Reed WD, Mooney RA, Lane MD 1975 Intracellular localization of the 3-hydroxy-3-methylglutaryl coenzyme A cycle enzymes in liver. J Biol Chem 250:3108-3116

6. Ayté J, Gil-Gómez G, Haro D, Marrero PF, Hegardt FG 1990 Rat mitochondrial and cytosolic 3-hydroxy-3-methylglutaryl-CoA synthases are encoded by two different genes. Proc Natl Acad Sci U S A 87:3874-3878

7. Thompson GN, Hsu BYL, Pitt JJ, Treacy E, Stanley CA 2071997 Fasting hypoketotic coma in a child with deficiency of mitochondrial 3-hydroxy-3-methylglutarylCoA synthase. N Engl J Med 337:1203-1201

8. Morris AAM, Lascelles CV, Olpin SE, Lake BD, Leonard JV, Quant PA 1998 Hepatic mitochondrial 3-hydroxy-3-methylglutaryl-CoA synthase deficiency. Pediatr Res 44:392-396

9. Boukaftane Y, Duncan A, Wang S, Labuda D, Robert M-F, Sarrazin J, Schappert K, Mitchell GA 1994 Human mitochondrial HMG CoA synthase (mHS): liver cDNA and partial genomic cloning, chromosome mapping to $1 \mathrm{p} 12-13$ and possible role in vertebrate evolution. Genomics 23:552-559

10. Hegardt FG 1995 Regulation of mitochondrial 3-hydroxy-3-methylglutaryl-CoA synthase gene expression in liver and intestine from the rat. Biochem Soc Trans 23:486-490

11. Boukaftane Y, Mitchell GA 1997 Cloning and characterization of the human mitochondrial 3-hydroxy-3-methylglutaryl CoA synthase gene. Gene 195:121-126

12. Bouchard L, Boukaftane Y, Bétard C, Hudson TJ, Mitchell GA 1998 The human mitochondrial 3-hydroxy-3-methylglutaryl CoA synthase gene (HMGCS2): characterization of an intragenic microsatellite (D1S3752) and gene mapping with radiation hybrids. Hum Mutat 12:291

13. Michaud J, Brody LC, Steel G, Fontaine G, Martin LS, Valle D, Mitchell GA 1992 Strand-separating conformational polymorphism (SSCP) analysis: efficacy of detection of point mutations in the human ornithine- $\delta$-aminotransferase gene. Genomics 13:389-394

14. Mitchell GA, Ozand PT, Robert M-F, Ashmarina L, Roberts J, Gibson M, Wanders RJ, Wang SP, Chevalier I, Plöchl E, Miziorko H 1997 HMG CoA lyase deficiency: identification of five causal point mutations in codons 41 and 42 , including a frequent Saudi Arabian mutation, R41Q. Am J Hum Genet 62:295-300

15. Van Dyk TK, Gatenby AA, LaRossa RA 1989 Demonstration by genetic suppression of interaction of GroE products with many proteins. Nature 342:451-453

16. Goloubinoff P, Gatenby AA, Lorimer GH 1999 GroE heat-shock proteins promote assembly of foreign prokaryotic ribulose bisphosphate carboxylase oligomers in Escherichia coli. Nature 337:44-47

17. Miziorko HM 1985 3-Hydroxy-3-methyglutaryl-CoA synthase from chicken liver. Methods Enzymol 110:19-26

18. Bradford MM 1976 A rapid and sensitive method for the quantitation of microgram quantities of protein utilizing the principle of protein-dye binding. Anal Biochem $72: 248-254$

19. Wang SP, Robert M-F, Gibson KM, Wanders RJA, Mitchell GA 1996 3-Hydroxy3-methylglutaryl-CoA lyase (HL): mouse and human HL gene (HMGCL) cloning and detection of large gene deletions in two unrelated HL-deficient patients. Genomics 33:99-104

20. Misra I, Narasimhan C, Miziorko HM 1993 Avian 3-hydroxy-3-methylglutaryl-CoA synthase - characterization of a recombinant cholesterogenic isozyme and demonstration of the requirement for a sulfhydryl functionality in formation of the acetylenzyme reaction intermediate. J Biol Chem 268:12129-12135

21. Mitchell GA, Fukao T 2001 Inborn errors of ketone body metabolism. In: Scriver CR, Beaudet AL, Sly WS, Valle D, Childs B, Vogelstein B (eds) The Metabolic and Molecular Bases of Inherited Disease. McGraw Hill, New York, pp 2327-2356 